\title{
Antiandrogen flutamide affects folliculogenesis during fetal development in pigs
}

\author{
Katarzyna Knapczyk-Stwora, Malgorzata Durlej-Grzesiak, Renata E Ciereszko", \\ Marek Koziorowski ${ }^{2,3}$ and Maria Slomczynska \\ Department of Endocrinology, Institute of Zoology, Jagiellonian University, Gronostajowa 9, 30-387 Krakow, Poland, \\ ${ }^{1}$ Department of Animal Physiology, University of Warmia and Mazury in Olsztyn, Oczapowskiego 1A, 10-718 \\ Olsztyn, Poland, ${ }^{2}$ Department of Physiology and Reproduction of Animals and ${ }^{3}$ Centre of Applied Biotechnology and \\ Basic Sciences, University of Rzeszow, Werynia 502, 36-100 Kolbuszowa, Poland \\ Correspondence should be addressed to M Slomczynska; Email: maria.slomczynska@uj.edu.pl
}

\begin{abstract}
Androgen deficiency during prenatal development may affect the expression of genes involved in the folliculogenesis regulation. In order to study the effect of antiandrogen on fetal ovarian development, pregnant gilts were injected with flutamide (for $7 \mathrm{days}, 50 \mathrm{mg} / \mathrm{kg}$ body weight per day) or corn oil (control groups) starting on gestation days 43 (GD50), 83 (GD90), or 101 (GD108). The obtained fetal ovaries were fixed for histology and immunohistochemistry or frozen for real-time PCR. Morphological evaluation, TUNEL assay, and expression of selected factors (Ki-67, GATA binding transcription factor 4 (GATA4), E-Cadherin and tumor necrosis factor $\alpha$ (TNF $\alpha$ )) were performed. On GD90 and GD108, ovaries following flutamide administration showed a higher number of egg nests and lower number of follicles than those in respective control groups. An increased mRNA and protein expression of Ki-67 was observed in flutamide-treated groups compared with controls on GD50 and GD108 but decreased expression was found on GD90. In comparison to control groups a higher percentage of TUNEL-positive cells was shown after flutamide exposure on GD50 and GD90 and a lower percentage of apoptotic cells was observed on GD108. These data were consistent with changes in TNF(TNF $\alpha$ ) mRNA expression, which increased on GD90 and decreased on GD108. E-cadherin mRNA and protein expression was upregulated on GD50 and downregulated on GD90 and GD108. In conclusion diminished androgen action in porcine fetal ovaries during mid- and late gestation leads to changes in the expression of genes crucial for follicle formation. Consequently, delayed folliculogenesis was observed on GD90 and GD108. It seems however that androgens exhibit diverse biological effects depending on the gestational period.
\end{abstract}

Reproduction (2013) $\mathbf{1 4 5} 265-276$

\section{Introduction}

The reproductive health of female farm animals is affected by a number of factors including endocrinedisrupting chemicals (EDC). Mammals are more susceptible to EDCs during fetal and neonatal life than in adulthood because of underdeveloped reproductive axes (Sweeney 2002). Environmental chemicals with androgenic and/or antiandrogenic activities are capable of interfering with androgen receptors (ARs) and regulate function of androgens within the endocrine system (Uzumcu \& Zachow 2007).

Androgens play an important role in ovarian development and functions, acting via ARs which are transcription factors (Drummond 2006). They are involved in the regulation of the expression of many target genes, which are significant during early stages of fetal folliculogenesis and are critical for reproduction (Nightingale et al. 2003). Previously, we demonstrated the presence of ARs in the porcine fetal ovary at different stages of gestation
(Burek et al. 2007) indicating the possible sites of androgen action. Moreover cytochrome P450c17 was found in oocyte nests and granulosa cells of primary follicles in porcine fetal ovaries (Knapczyk-Stwora et al. 2011) suggesting local androgen production during early development.

In many mammals, including pigs, folliculogenesis begins during fetal development and continues throughout adulthood (Bielanska-Osuchowska 2006). The morphological differentiation of the porcine ovary is initiated around day 33 of the prenatal period and is characterized by the formation of medullary cords, which degenerate by gestational day 44 (McCoard et al. 2001). The formation of ovarian follicles starts on day 56 of gestation, while follicles surrounded by a single layer of somatic cells are present on gestational day 106 . The assembly of primordial follicles and their subsequent transition to the primary stage occur in late gestational and neonatal period (Bielanska-Osuchowska 2006). 
The proper development of an ovary during fetal life is essential for fertility and reproductive success in adulthood (for review see Sarraj \& Drummond 2012). Therefore, any disorders during fetal ovarian differentiation may result in incomplete sexual development or cause infertility (de Boo \& Harding 2006). The breakdown of egg nests (EN) and the formation of primordial follicles represent a critical stage in ovarian development. Follicular assembly is associated with the apoptotic breakdown of germ cells within nests. This apoptosis is coordinated by apoptotic factors such as tumor necrosis factor $\alpha$ (TNF $\alpha$; Morrison \& Marcinkiewicz 2002, Aitken et al. 2011). Furthermore various intraovarian factors such as transcription factors and growth factors are involved in the regulation of early follicle formation (Epifano \& Dean 2002). A very early marker of gonad formation is GATA-binding protein 4 (GATA4), the zinc finger transcription factor, involved in gonadal development in many species (McCoard et al. 2001). Androgens are known to modulate the expression of cell-cell adhesion molecules including E-cadherin in a tissuespecific manner (Carruba et al. 1995). E-cadherin plays a crucial role during somatic cell and oocyte assembly forming primordial follicles (Wang \& Roy 2010).
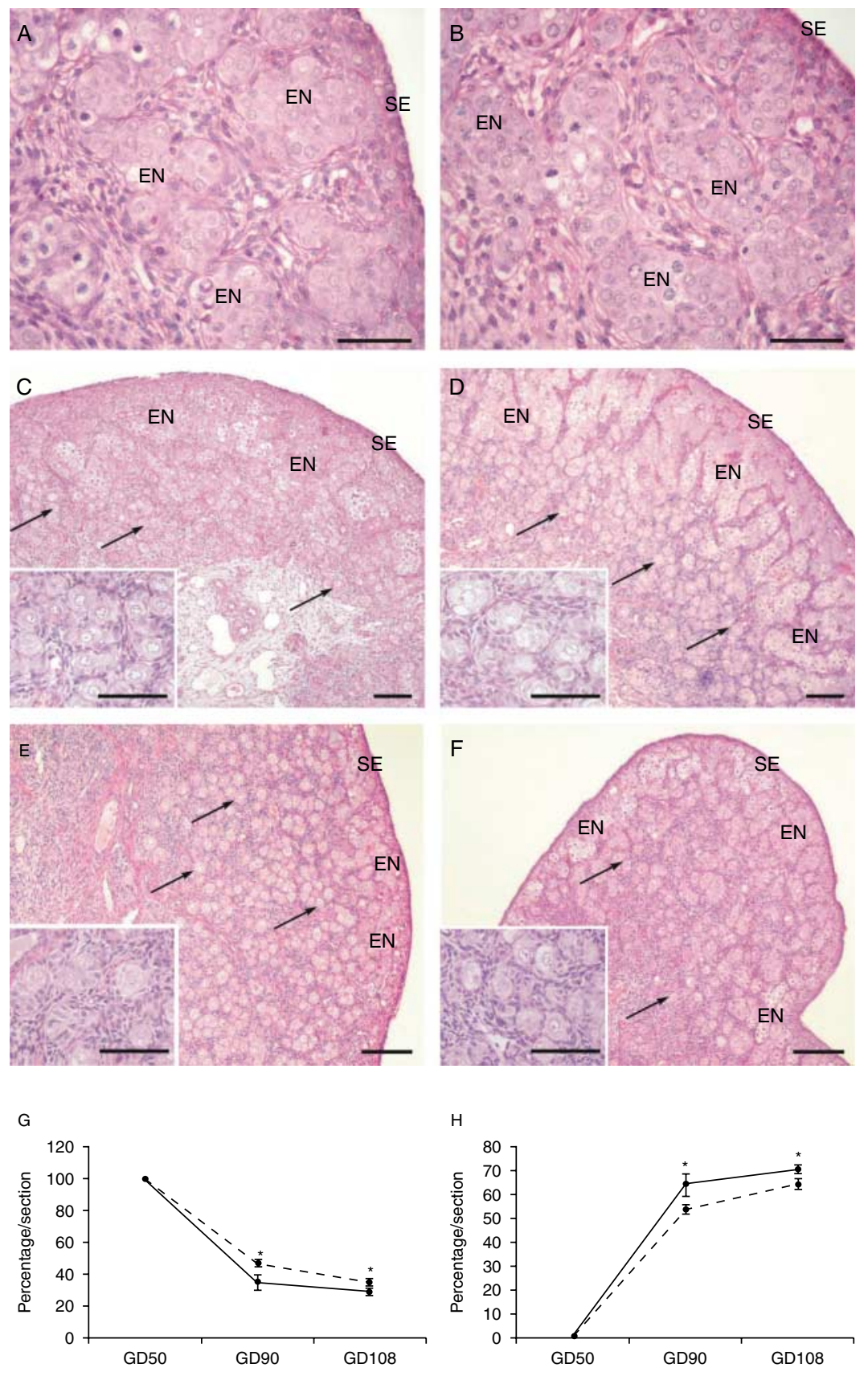

Figure 1 Histoarchitecture of the fetal ovaries obtained on days 50 (A and $B), 90(C$ and $D)$, and 108 ( $E$ and $F$ ) of gestation from control $(A, C$, and $E$ ) and flutamide-exposed (B, D, and F) porcine fetuses using H\&E staining. Graphs represent percentages (means \pm s.E.M.) of EN (G) and follicles (H) per section in the examined fetal ovaries. Values represent means \pm S.E.M. Asterisks denote significant differences (Mann-Whitney $U$ test; $P<0.05$ ). EN, egg nests; $S E$, surface epithelium; arrows, forming follicles; GD50, flutamide administered daily on gestation days 43-49; GD90, flutamide administered daily on gestation days 83-89; GD108, flutamide administered daily on gestation days 101-107. Scale bars represent: $50 \mu \mathrm{m}$ (A, B and all insets) $100 \mu \mathrm{m}$ (C, D, E and F). 
Therefore, it seems possible that androgen deficiency during fetal life may affect androgen-dependent gene expression and disturb the normal process of folliculogenesis. Flutamide, a non-steroid antiandrogen, binds to and blocks androgen receptors (ARs) (Tevell et al. 2006). As we showed previously flutamide applied in utero on critical days of gestation alters expression of androgendependent genes during postnatal life (Durlej et al. 2011a, 2011b). In light of our previously published results, which demonstrated the presence of $A R$ in the fetal porcine ovaries (Burek et al. 2007), we hypothesized that limited access to androgens may have an impact on the expression of some intraovarian factors responsible for fetal folliculogenesis. The aim of this study was to examine the effect of flutamide on fetal ovarian development in pigs. To meet this goal morphological evaluation of the ovary, cell proliferation (Ki-67) and apoptosis, as well as mRNA (Ki-67, GATA4, E-cadherin and TNF $\alpha$ ) and protein (GATA4 and E-cadherin) expression of selected factors, were performed using real-time PCR and immunohistochemistry.

\section{Results}

\section{Effect of flutamide on fetal ovarian morphology and} proportion of EN and follicles

Representative images of fetal porcine ovaries at each developmental stage are shown in Fig. 1. On gestation day 50 (GD50), naked eggs located in nests (EN) were present within the fetal ovarian cortex of control and flutamidetreated animals (Fig. 1A and B). Ovarian follicles were not observed on GD50 both in control and in flutamidetreated animals. Apart from EN observed within the outer ovarian cortex under the surface epithelium (SE) the forming follicles (arrows) were found in the inner cortex (Fig. 1C, D, E, and F insets) on days 90 (Fig. 1C and D) and 108 (Fig. 1E and F). On GD90 and GD108, fetal ovaries following flutamide administration displayed a higher $(P<0.05)$ number of EN (Fig. $1 \mathrm{G})$ and lower $(P<0.05)$ number of follicles (Fig. $1 \mathrm{H}$ ) when compared with the respective control groups.

\section{Effect of flutamide on MKI67 (Ki-67), TNF, GATA4 and CDH1 (E-cadherin) mRNA expression in fetal ovaries}

On GD50 (Fig. 2A) flutamide treatment caused upregulation of $\mathrm{CDH} 1$ expression $(P<0.01)$. On GD90 (Fig. 2B) flutamide administration resulted in an increased expression of TNF mRNA $(P<0.05)$ and decreased expression of GATA4 $(P<0.01)$ and $C D H 1$ $(P<0.01)$ mRNAs. No changes were observed in MKI67 expression. On GD108 (Fig. 2C) MKI67 mRNA was upregulated $(P<0.05)$ but $T N F$ and $C D H 1$ mRNAs were downregulated $(P<0.05$ and $P<0.01$ respectively) when compared with the control group. The level of
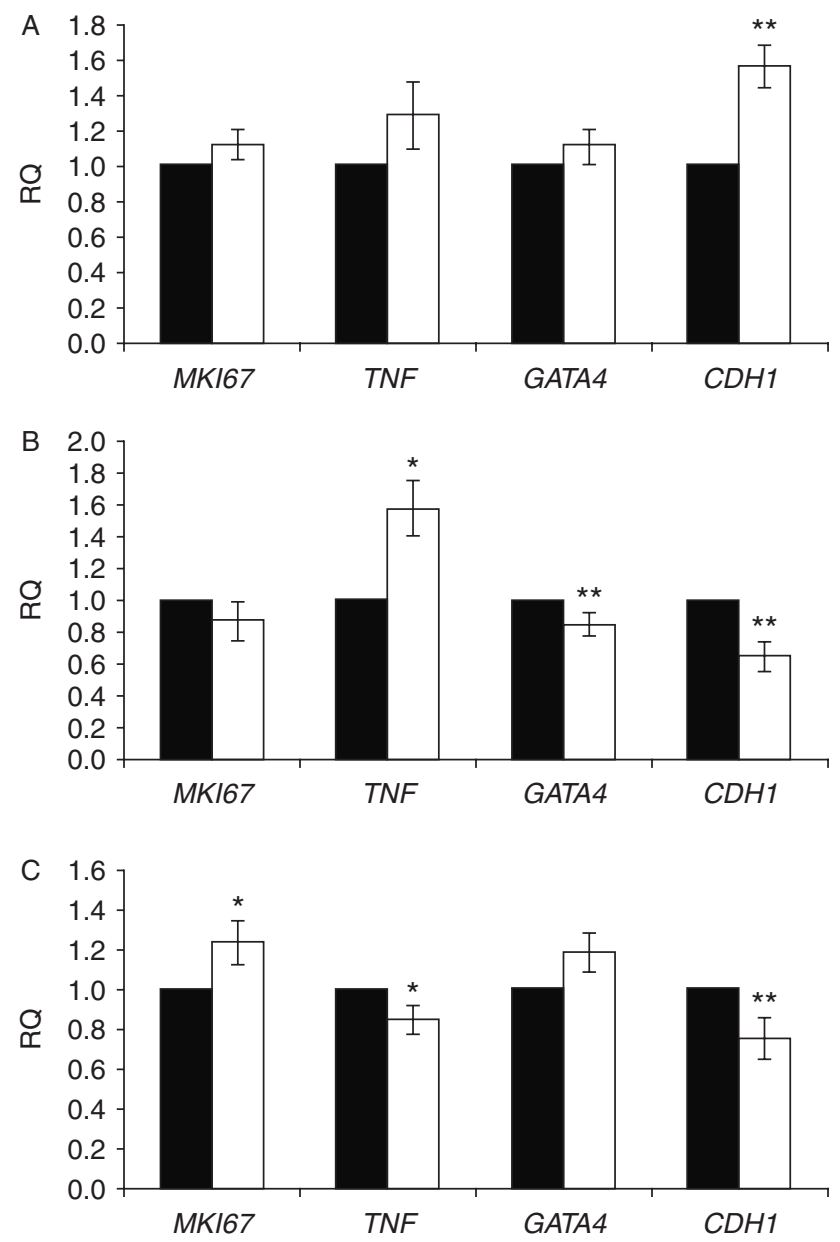

Figure $2 M K 167, T N F, G A T A 4$, and $C D H 1$ mRNA expression in porcine ovaries obtained on days 50 (A), 90 (B) and 108 (C) of gestation from control and flutamide-exposed fetuses. Relative expression of MKI67, TNF, GATA4 and CDH1 mRNAs was determined with the use of quantitative real-time PCR analysis. Relative quantification (RQ) is expressed as mean \pm s.E.M. (shaded bars, control groups; open bars, flutamide-treated groups). $\Delta C$ t values were used to find statistical differences using the Mann-Whitney $U$ test. Asterisks denote statistically significant differences $\left({ }^{*} P<0.05,{ }^{* *} P<0.01\right)$.

GATA4 mRNA was not significantly changed after GD108 flutamide administration.

\section{Effect of flutamide on proliferation and apoptosis in fetal ovaries}

The percentage of proliferating cells was assessed using immunohistochemical determination of $\mathrm{Ki}-67$ expression (Fig. 3A, B, C, D, E and F). In all examined sections positive Ki-67 nuclear staining was observed only in germ cells within EN (open arrowheads), while no positive staining was found in somatic cells. The percentage of apoptotic cells on the examined days of gestation was determined using TUNEL assay (Fig. 4A, B, C, D, E and F). In situ detection of DNA fragmentation 

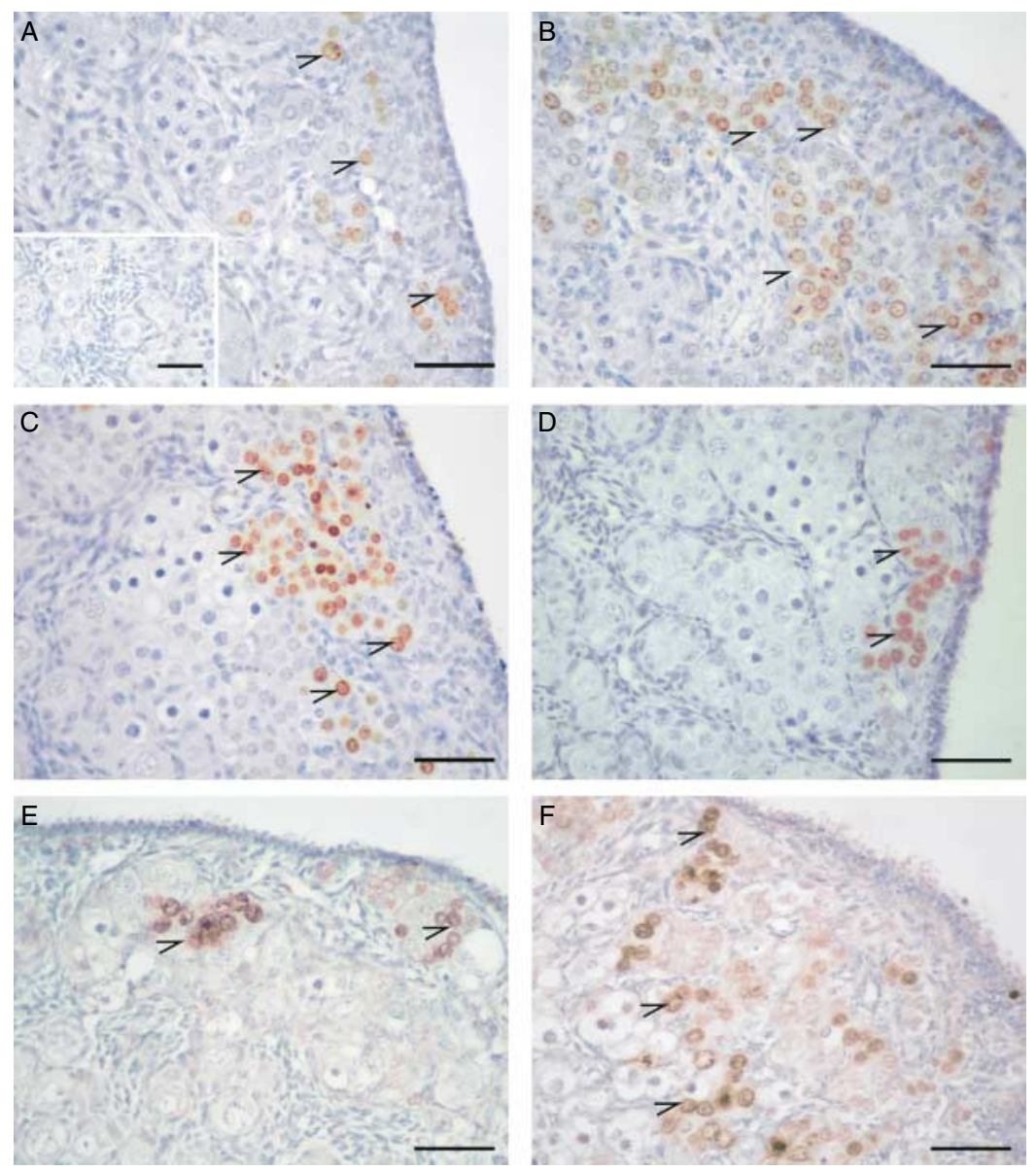

Figure 3 Localization of Ki-67 (a proliferation marker) in the fetal ovaries obtained on days 50 (A and B), 90 $(\mathrm{C}$ and $\mathrm{D})$ and 108 (E and F) of gestation from control and flutamide-exposed fetuses. Open arrowheads are pointing at oogonia/oocytes showing a positive Ki-67 immunostaining (A, B, C, D, E and F). Control sections in which the primary antibody against Ki-67 was replaced by mouse IgG did not exhibit any positive staining ( $A$, inset). GD50, flutamide administered daily on gestation days 43-49; GD90, flutamide administered daily on gestation days 83-89; GD108, flutamide administered daily on gestation days 101-107. All the scale bars represent $50 \mu \mathrm{m}$. revealed single apoptotic germ cells displaying brown staining in their nuclei (open arrowheads).

A significant increase $(P<0.001)$ in the percentage of Ki-67-positive cells (Table 1 and Fig. $3 \mathrm{~B}$ ) and in the percentage $(P<0.001)$ of TUNEL-positive cells (Table 1 and Fig. 4B) was found on GD50 following flutamide treatment when compared with controls (Figs $3 \mathrm{~A}$ and 4A respectively). On GD90 flutamide administration resulted in diminished proliferation $(P<0.001$; Table 1 and Fig. 3D) and elevated apoptosis $(P<0.001$; Table 1 and Fig. 4D) in comparison to control groups (Figs 3C and $4 \mathrm{C}$ respectively). On GD108 the percentage of $\mathrm{Ki}-67$-immunopositive cells was significantly increased $(P<0.001$; Table 1 and Fig. 3F), whereas the percentage of apoptotic cells was significantly diminished $(P<0.01$; Table 1 and Fig. 4F) following flutamide exposure compared with control groups (Figs $3 \mathrm{E}$ and $4 \mathrm{E}$ respectively).

\section{Effect of flutamide on GATA4 immunoexpression in fetal ovaries}

In all examined sections, positive GATA4 nuclear staining was observed only in somatic cells, whereas germ cells did not exhibit any staining (Fig. 5A, B, C, D,
E and F). On GD50, GATA4 was localized in stroma cells surrounding EN and there were no significant differences in the staining intensity when compared with the control group (Fig. 5A, B and G). On GD90 and GD108 in control (Fig. 5C and $\mathrm{E}$ respectively) and flutamide-treated (Fig. 5D and F respectively) groups GATA4 was present in outer (stroma cells surrounding EN) and inner (granulosa cells of forming follicles and stromal cells around follicles) cortex. However, GATA4 immunoreaction was lower in both outer and inner ovarian cortex $(P<0.05)$ of the flutamide-treated group than in the control group only on GD90 (Fig. 5G).

\section{Effect of flutamide on E-cadherin immunoexpression in fetal ovaries}

In all examined sections, positive E-cadherin cytoplasmic staining was observed in EN (Fig. 6A, B, C, D, $\mathrm{E}$ and $\mathrm{F}$ ). Additionally E-cadherin was localized in oocytes' cytoplasm of forming follicles on GD90 (Fig. 6C and D insets) and GD108 (Fig. 6E and F insets). On GD50 flutamide administration resulted in increased $(P<0.05)$ E-cadherin expression when compared with the control group (Fig. 6G). In contrast on GD90 and GD108 decreased expression of E-cadherin was 

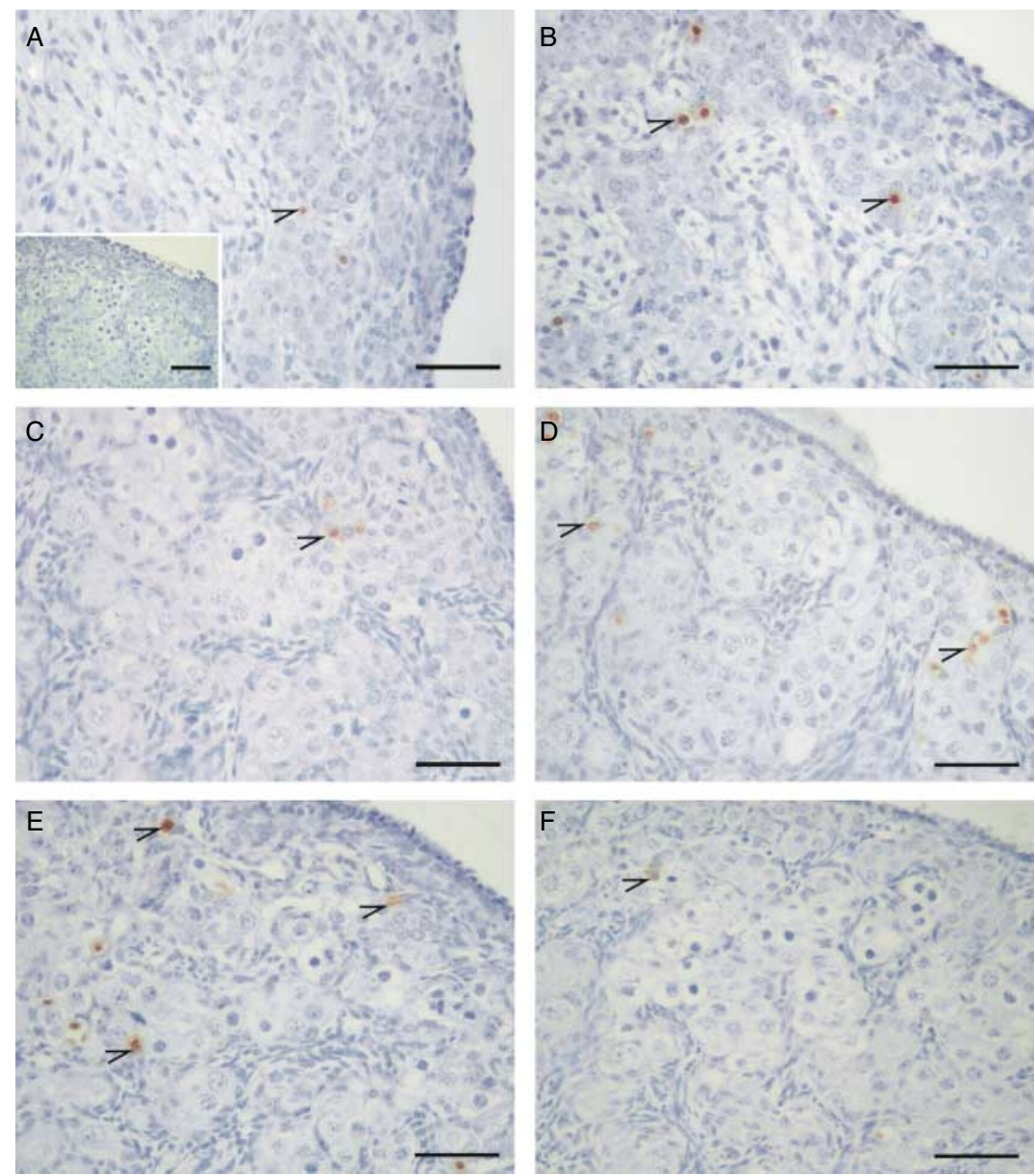

Figure 4 Localization of apoptotic cells in the fetal ovaries obtained on days 50 (A and B), 90 (C and D) and 108 ( $E$ and $F$ ) of gestation from control and flutamide-exposed fetuses. Apoptotic cells were identified using TUNEL. Open arrowheads indicate single apoptotic oogonia/oocytes (A, B, C, D, E and $\mathrm{F})$. A negative control for TUNEL assay was performed without active TdT enzyme (A, inset). GD50, flutamide administered daily on gestation days 43-49; GD90, flutamide administered daily on gestation days 83-89; GD108, flutamide administered daily on gestation days $101-107$. All the scale bars represent $50 \mu \mathrm{m}$. observed in EN $(P<0.01, P<0.05$ respectively $)$ and oocytes of forming follicles $(P<0.001, P<0.01$ respectively) in comparison to controls (Fig. 6G).

\section{Discussion}

The role of androgens in the ovaries of adult animals was reported previously and was well reviewed by Walters et al. (2008). Much less data are available on AR-mediated effects during fetal development. Ovarian formation and folliculogenesis begin early during fetal development and continue after birth. In rodents, primordial follicle formation begins in early postnatal life, while in humans and domestic animals, this process is observed during fetal life (for review see Sarraj \& Drummond 2012). Therefore we focused our attention on the role of androgens within a critical window of porcine fetal ovarian development.

In prenatal testosterone-treated sheep, the consequences of elevated androgen level (polycystic ovarian morphology, enhanced follicular recruitment/depletion and increased estradiol secretion) were observed in

Table 1 Effect of prenatal flutamide administration on proliferation (Ki-67 expression) and apoptosis (TUNEL assay) rates in the porcine fetal ovaries on days 50, 90 and 108 of gestation.

\begin{tabular}{|c|c|c|c|c|c|c|}
\hline \multirow{2}{*}{$\begin{array}{l}\text { Percentage of } \\
\text { positive cells in egg } \\
\text { nests/section }\end{array}$} & \multicolumn{2}{|c|}{ GD50 } & \multicolumn{2}{|c|}{ GD90 } & \multicolumn{2}{|c|}{ GD108 } \\
\hline & Control & Flutamide & Control & Flutamide & Control & Flutamide \\
\hline $\begin{array}{l}\text { Ki-67 } \\
\text { TUNEL }\end{array}$ & $\begin{array}{c}25.74 \pm 1.2 \\
1.13 \pm 0.27\end{array}$ & $\begin{array}{r}45.28 \pm 1.88^{+} \\
3.47 \pm 0.49^{+}\end{array}$ & $\begin{array}{r}21.86 \pm 1.86 \\
1.07 \pm 0.33\end{array}$ & $\begin{array}{r}14.17 \pm 0.91^{+} \\
3.74 \pm 0.33^{+}\end{array}$ & $\begin{array}{r}13.25 \pm 1.8 \\
2.7 \pm 0.88\end{array}$ & $\begin{array}{r}22.97 \pm 1.96^{+} \\
0.67 \pm 0.18^{*}\end{array}$ \\
\hline
\end{tabular}

Values are expressed as means \pm S.E.M. Asterisks denote significant differences between control and flutamide-treated animals within each gestational group (Mann-Whitney $U$ test; ${ }^{*} P<0.01,{ }^{+} P<0.001$ ). GD50, flutamide administration between days 43 and 49 of gestation; GD90, flutamide administration between days 83 and 89 of gestation; GD108, flutamide administration between days 101 and 107 of gestation. 

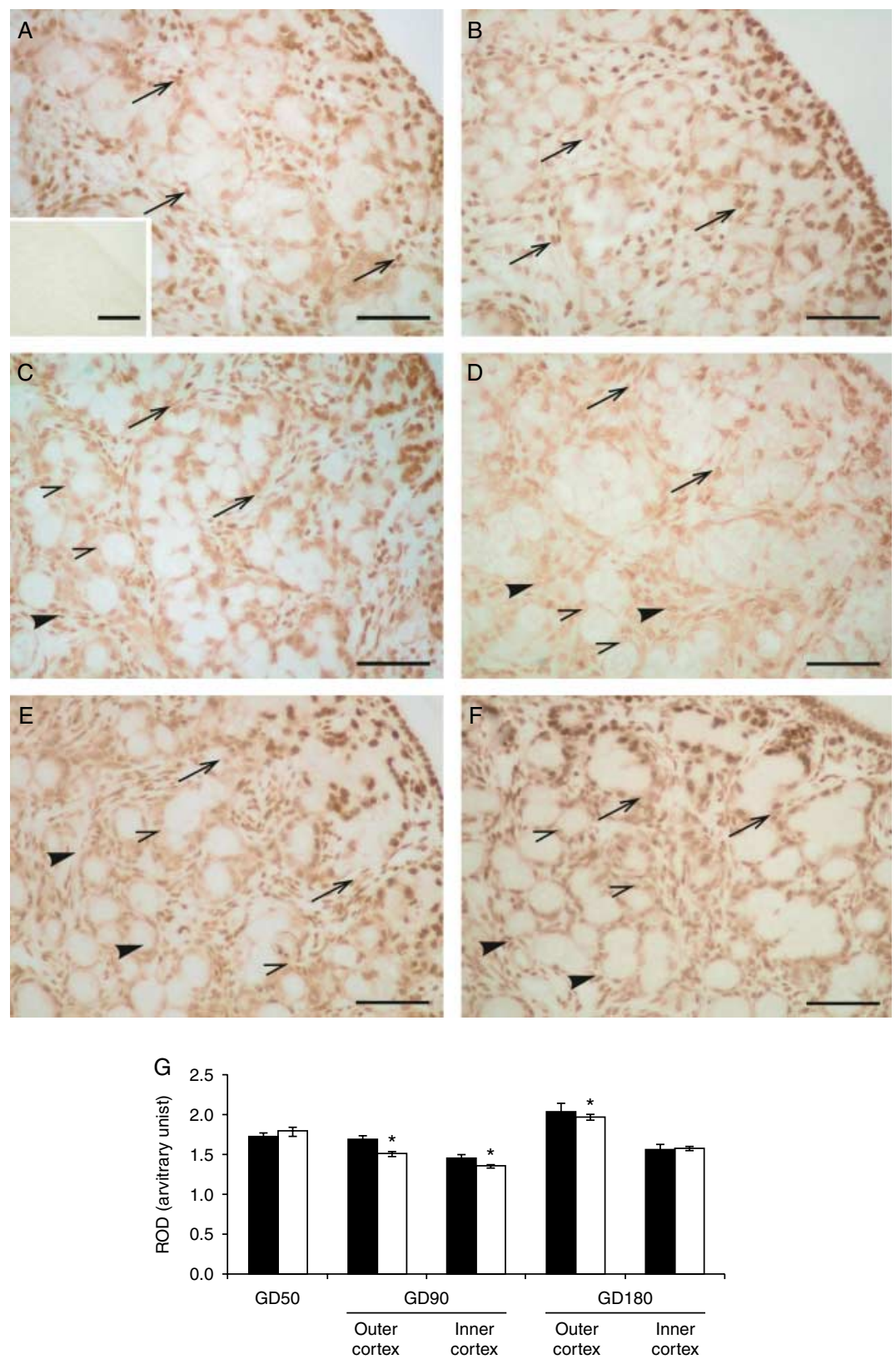

Figure 5 GATA4 immunostaining in the fetal porcine ovaries obtained from control $(\mathrm{A}, \mathrm{C}$ and $\mathrm{E})$ and flutamide-exposed (B, D and $F$ ) fetuses on days 50 $(A$ and $B), 90(C$ and $D)$ and 108 ( $E$ and $F)$ of gestation. In all representative micrographs arrows indicate positive GATA4 staining in stroma cells surrounding egg nests within outer ovarian cortex. Within inner ovarian cortex, GATA4-positive staining was found in granulosa cells of forming follicles (open arrowheads) and in stromal cells around follicles (arrowheads) from both control and flutamide-exposed fetuses on days 90 (C and D respectively) and 108 ( $E$ and $F$ respectively) of gestation. Control sections in which the primary antibody was replaced by goat IgG did not exhibit any positive staining (A, inset). Charts $(G)$ represent the intensity of GATA4 immunostaining expressed as a relative optical density (ROD) in the fetal porcine ovaries for all examined days (shaded bars, control groups; open bars, flutamide-treated groups). Bars represent means \pm S.E.M. Asterisks denote significant differences (Mann-Whitney $U$ test; ${ }^{*} P<0.05$ ).

GD50, flutamide administered daily on gestation days 43-49; GD90, flutamide administered daily on gestation days 83-89; GD108, flutamide administered daily on gestation days 101-107. All the scale bars represent $50 \mu \mathrm{m}$. adulthood (Steckler et al. 2007). The excess of prenatal androgens disrupted the expression of ovarian steroid receptor protein, altering the ovarian developmental trajectory and causing an imbalance in AR/estrogen receptor (ER) protein in sheep (Ortega et al. 2009). Moreover, female rhesus monkeys (Abbott et al. 1998), sheep (Steckler et al. 2007), mice (Sullivan \& Moenter 2004) and rats (Foecking et al. 2005) prenatally exposed to either testosterone or dihydrotestosterone (DHT) exhibited disorders in the ovulatory cycles in adulthood. The androgen excess phenotypes resemble those of women with polycystic ovary syndrome (PCOS), suggesting a fetal origin of PCOS (Abbott et al. 2002).
Thus, the fetal androgen excess has striking consequences for adult ovarian functions.

Androgen deficiency is another approach for investigating cellular and molecular mechanisms of androgen action. Animal models and antiandrogen treatment are frequently applied in this approach. The administration of flutamide to pregnant rhesus monkeys induced androgen deficiency during the critical gestation window. Our previous studies on flutamide-treated pigs revealed that prenatal and neonatal changes in androgen actions resulted in altered expression of androgen-dependent genes in adulthood (Durlej et al. $2011 b$, 2012). To date most studies concerning the 
involvement of androgens in the process of fetal gonadal development were focused on examination of postnatal life. The current study was undertaken to show which genes responsible for folliculogenesis are affected by androgen deficiency during the critical window of gestation. Therefore we used ovaries obtained from female fetuses at different stages of development. We hypothesized that exposure to the AR antagonist flutamide during fetal development disrupts androgen signaling and results in impaired ovarian development. In this way androgen deficiency may be partially responsible for some of the animals' postnatal reproductive features. To our knowledge this is the first study investigating the interactions between androgens and locally expressed factors involved in development of fetal ovaries.

In the study flutamide-treated ovaries on GD90 and GD108 exhibited a higher number of EN and lower number of follicles when compared with the respective controls. It maybe assumed that the exposure to flutamide led to the delayed follicle formation in fetal porcine ovaries. The obtained results strongly support the notion that androgens promote growth of preantral follicles (Vendola et al. 1998, Forsdike et al. 2007). It was reported previously that testosterone and DHT acting via $A R$ increased the number of primordial
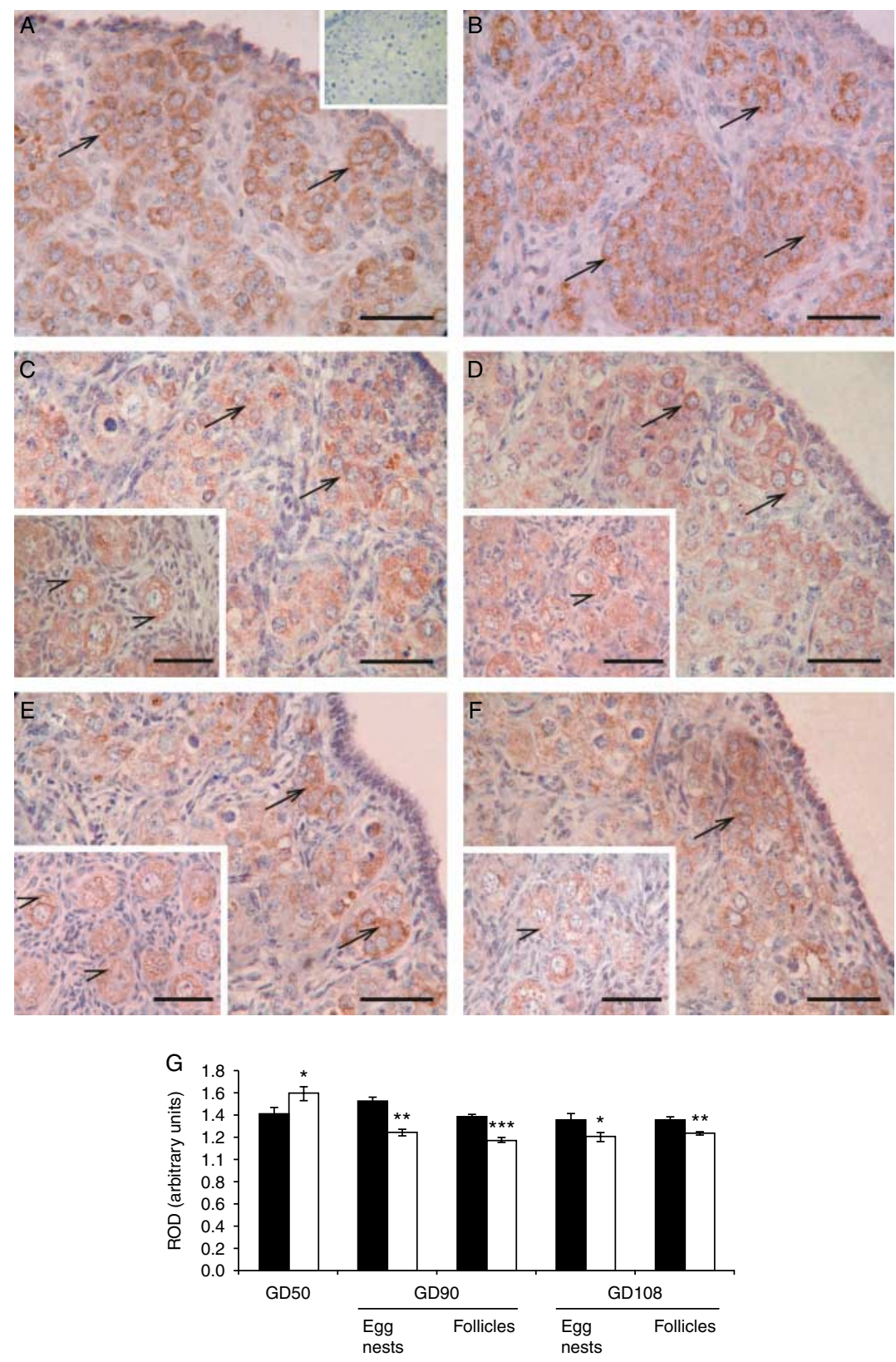

Figure 6 E-cadherin immunostaining in the fetal porcine ovaries obtained on days 50 (A and B), 90 $(C$ and $D)$ and 108 (E and $F$ ) of gestation from control (A, C and E) and flutamide-exposed (B, D and $F$ ) fetuses. In all representative micrographs arrows indicate positive E-cadherin staining in egg nests. E-cadherin immunostaining was found in the oocytes of forming follicles (open arrowheads) from both control and flutamide-exposed fetuses on days 90 (C inset and D inset respectively) and 108 (E inset and $\mathrm{F}$ inset respectively) of gestation. Control sections in which the primary antibody was replaced by rabbit IgG did not exhibit any positive staining $(A$, inset). Charts $(G)$ represent the intensity of E-cadherin immunostaining expressed as a relative optical density (ROD) in the fetal porcine ovaries for all examined days (shaded bars, control groups; open bars, flutamide-treated groups). Bars represent means \pm S.E.M. Asterisks denote significant differences (Mann-Whitney $U$ test; ${ }^{*} P<0.05$, $\left.{ }^{* *} P<0.01, * * * P<0.001\right)$. GD50, flutamide administered daily on gestation days 43-49; GD90, flutamide administered daily on gestation days 83-89; GD108, flutamide administered daily on gestation days 101-107. All the scale bars represent $50 \mu \mathrm{m}$. 
follicles in mice (Yang et al. 2010). It was also shown that androgens promoted activation of primordial follicle recruitment by $\mathrm{FOXO} 3$ phosphorylation and its translocation into the nucleus. As FOXO3 was identified in porcine fetal and neonatal ovaries (Ding et al. 2010), it is possible that $\mathrm{FOXO} 3$ inactivation resulting from the inhibition of androgen action was partially responsible for the delayed folliculogenesis.

In the current study, we showed a higher percentage of apoptotic cells after flutamide exposure on GD50 and GD90 and a lower percentage of apoptotic cells on GD108. These results are consistent with the changes in $T N F(T N F \alpha)$ mRNA expression. It is known that TNF $\alpha$ is important in promoting the oocyte apoptosis that accompanies oocyte nest breakdown and follicle assembly (Morrison \& Marcinkiewicz 2002). It should also be emphasized that fetal ovarian development in pigs involves both apoptosis and proliferation (Garrett \& Guthrie 1999). Examination of the incidence of proliferation revealed higher mRNA and protein expression of Ki-67 following flutamide administration on GD50 and GD108 but lower on GD90. Therefore it appears that androgen deficiency during pregnancy affects the balance between apoptosis and proliferation in fetal porcine ovary.

In fetal ovaries on GD90 we observed a decrease in GATA4 mRNA and protein expression as well as in proliferation following flutamide administration. In pigs, GATA4 has been demonstrated from the earliest stages of gonadogenesis (McCoard et al. 2001). However, the target genes for GATA4 are still unknown. Heikinheimo et al. (1997) reported abundant GATA4 expression during proliferation of granulosa cells of primary and early antral follicles. On the other hand the lack of significant differences in GATA4 expression between treated and nontreated animals on GD50 and GD108 does not support GATA4 involvement in the regulation of cell proliferation in fetal porcine ovaries during the examined periods.

In this study we demonstrated increased E-cadherin mRNA and protein expression in the flutamide-treated group on GD50. E-cadherin was found in the fetal ovary of hamsters (Wang \& Roy 2010), mice (Mackay et al. 1999), pigs (Ryan et al. 1996), and humans (Stoop et al. 2005). Androgens have been reported to modulate the expression of cell-cell adhesion molecules, including E-cadherin (Carruba et al. 1995). The increase in E-cadherin expression observed in our experiment might affect the normal process of EN breakdown. On the other hand the flutamide administration during late gestation (GD90 or GD108), i.e. during period of typically increased E-cadherin level (Ryan et al. 1996, Wang \& Roy 2010) resulted in a diminished E-cadherin expression in the fetal ovaries. This low level of E-cadherin should be observed earlier during gestation. A decrease in E-cadherin expression within the breakdown of EN coincided with the formation of primordial follicles (Mackay et al. 1999). It seems that a decline in E-cadherin expression is crucial for the formation of the primordial follicles. Therefore the observed flutamideinduced decrease in E-cadherin expression might be related to delayed folliculogenesis. It appears that misregulation of E-cadherin expression can alter ovarian development.

In the current study we demonstrated a higher number of EN and lower number of follicles in the flutamidetreated ovaries on GD90 and GD108 in pigs. We have also shown that the expression patterns of several genes critically involved in the differentiation of the porcine fetal ovary are affected by antiandrogen treatment in utero. We suggest that the changes in gene expression may be contributing to the delayed folliculogenesis. Androgen actions however may depend on the gestational period. It should also be emphasized that antiandrogen treatment may not completely block AR signaling and it may exert effects that are not associated with androgen signaling (Ing 2005). In summary our results suggest that in utero antiandrogenic exposure interferes with the expression of genes involved in fetal folliculogenesis.

\section{Materials and Methods}

\section{Experiment design and tissue collection}

Sexually mature crossbred gilts $(n=12$; Large White $\times$ Polish Landrace) of similar age (10-11 months) and body weight $(100-120 \mathrm{~kg})$ were used for this study. After exhibiting two estrous cycles of normal length, gilts were observed for estrus behavior twice daily and mated to fertile boars at the onset of estrus as well as 12 and $24 \mathrm{~h}$ later. The gestation day (GD) was estimated from the first mating day. Pregnant gilts were randomly allocated to three experimental groups: i) animals injected with flutamide (Sigma-Aldrich) between days 43 and 49 of gestation (GD50, $n=2$ ), ii) animals injected with flutamide between days 83 and 89 of gestation (GD90, $n=2$ ), and iii) animals injected with flutamide between days 101 and 107 of gestation (GD108, $n=2$ ). The days chosen for in utero exposure to flutamide are critical for fetal ovarian development in pig and include the period of EN formation (GD50), primordial follicle formation (GD90), and its transition to primary follicles (GD108) (Bielanska-Osuchowska 2006).

Flutamide was suspended in corn oil and administered by s.c. injections daily for 7 days at a dose of $50 \mathrm{mg} / \mathrm{kg}$ body weight. The flutamide dose was based on the literature data (Williams et al. 2001, Foster \& Harris 2005) and results of our previous studies (Durlej et al. 2011a, 2011b); however time of antiandrogen administration was extended and frequency was increased. For each flutamide-exposed group a respective control group was used ( $n=2$ per each gestation period) and control animals were treated with corn oil in a manner similar to the flutamide-treated pigs.

The female fetuses were obtained during surgery performed under thiopental anesthesia from flutamide-treated and control pregnant gilts on GD50 ( $n=9$ and $n=10$ respectively), on 
GD90 ( $n=14$ and $n=5$ respectively), or on GD108 ( $n=11$ and $n=6$ respectively). Ovaries of each female fetus were immediately excised: one ovary was fixed in Bouin's solution for routine histology (hematoxylin-eosin staining, H\&E) and immunohistochemistry, while the contralateral ovary was snap frozen in liquid nitrogen for RNA isolation.

All surgical procedures were performed by a veterinarian and followed approved guidelines for the ethical treatment of animals in accordance with the Polish legal requirements under the license given by the Local Ethics Committee at Jagiellonian University (No. 122/2009).

\section{RNA preparation and TaqMan real-time PCR analysis}

Total RNA was extracted from fetal ovaries using TRI Reagent solution (Ambion, Austin, TX, USA) following the manufacturer's instructions. RNA samples were electrophoresed on a $1 \%(\mathrm{w} / \mathrm{v})$ denaturating agarose gel to verify the RNA quality and were stored frozen at $-80{ }^{\circ} \mathrm{C}$. RNA concentration and purity were determined immediately before $\mathrm{RT}$ through measurement of $\mathrm{A}_{260}: \mathrm{A}_{280}$ ratios with a NanoDrop ND2000 Spectrophotometer (Thermo Scientific, Wilmington, DE, USA) and a volume equivalent to $1 \mu \mathrm{g}$ RNA was taken for RT. RNA samples were reverse transcribed using a High-Capacity cDNA Reverse Transcription kit (Applied Biosystems) according to the manufacturer's protocol. Reverse transcriptase reaction mixtures were prepared in a $20 \mu \mathrm{l}$ volume using random primers, dNTP mix, RNAse inhibitor and Multi Scribe reverse transcriptase. The RT was performed in a Veriti Thermal Cycler (Applied Biosystems) with a temperature cycling program of $10 \mathrm{~min}$ at $25^{\circ} \mathrm{C}, 2 \mathrm{~h}$ at $37{ }^{\circ} \mathrm{C}$ and $5 \mathrm{~min}$ at $85^{\circ} \mathrm{C}$, with subsequent cooling to $4^{\circ} \mathrm{C}$. Genomic DNA amplification contamination was checked periodically by control experiments in which reverse transcriptase was omitted during the RT step. Samples were kept at $-20{ }^{\circ} \mathrm{C}$ until further analysis.

Real-time PCR analyses were performed using the StepOne Real-Time PCR System (Applied Biosystems). The expression level of MKI67, TNF, GATA4 and CDH1 was quantified in each sample using TaqMan Gene Expression Assay (Applied Biosystems). GAPDH levels were used as endogenous control (for details see Table 2). Quantitative PCR was performed with 200 ng cDNA, $1 \mu$ l Gene Expression Assay and $10 \mu$ l TaqMan PCR master mix (Applied Biosystems) in a final volume of $20 \mu \mathrm{l}$. After a 2 -min incubation at $50{ }^{\circ} \mathrm{C}$, the thermal cycling conditions were $10 \mathrm{~min}$ at $95^{\circ} \mathrm{C}$ followed by 40 repeats of $15 \mathrm{~s}$ at $95{ }^{\circ} \mathrm{C}$ and $1 \mathrm{~min}$ at $60{ }^{\circ} \mathrm{C}$ to determine the cycle threshold $(C t)$ for quantitative measurement. Relative quantification (RQ) was obtained using the $2^{-\Delta \Delta C t}$ method, adjusting the MKI67, TNF, GATA4 and CDH1 expression to the expression of $G A P D H$ and considering the adjusted expression in the control samples as reference $(R Q=1)$ (Livak \& Schmittgen 2001). Data were expressed as mean $\mathrm{RQ} \pm$ s.E.M. For calculating the statistical significance of differences in examined genes' expression between controls and corresponding flutamidetreated groups, all samples were normalized to the GAPDH ( $\Delta C$ t value). These $\Delta C$ t values were used to find statistical differences by means of the Mann-Whitney $U$ test.

\section{General morphology}

The tissue sections $(5 \mu \mathrm{m})$ were dewaxed in xylene, rehydrated in ethanol and rinsed in water. To stain basophilic structures, including the nuclei, the sections were placed in hematoxylin for $35 \mathrm{~s}$. Next the sections were washed in tap water to increase the blue color and dehydrated in ethanol. The cytoplasm of cells was then stained pink with alcoholic solution of eosin $Y$ for $10 \mathrm{~s}$. Sections were then washed in ethanol, fixed in xylene and mounted using DPX (Fluka Chemie $\mathrm{GmbH}$, Buchs) and coverslip.

On GD90 and GD108, the number of particular ovarian structures (follicles and EN) was counted in five sections (the middle cross section and four other sections) per ovary. Proportion of examined ovarian structures was calculated as the percentage of EN or follicles on the number of total EN and follicles per section (Ding et al. 2010). The counting was performed in each field using a microscope with a $\times 20$ objective.

\section{Immunohistochemistry}

The $5 \mu \mathrm{m}$-thick ovarian sections were mounted on $3^{\prime} 3^{\prime}$ aminopropyl-triethoxysaline (Sigma-Aldrich)-coated slides. Then they were deparaffinized in xylene, rehydrated gradually through a series of ethanol dilutions and rinsed in water. Next the sections were immersed in $0.01 \mathrm{M}$ citrate buffer $(\mathrm{pH} 6.0)$ and heated in a microwave oven $(3 \times 4 \mathrm{~min}, 600 \mathrm{~W})$ to retrieve antigen. Endogenous peroxidase activity was prevented by incubation with $0.3 \%(\mathrm{v} / \mathrm{v}) \mathrm{H}_{2} \mathrm{O}_{2}$ in Tris-buffered saline (TBS; $0.1 \mathrm{M}$ Tris and $150 \mathrm{mM} \mathrm{NaCl}, \mathrm{pH}$ 7.4). Blocking of nonspecific binding sites was performed with $5 \%(\mathrm{v} / \mathrm{v})$ normal horse (for Ki-67 and GATA4) or goat (for E-cadherin) serum (Sigma-Aldrich) before incubation with primary antibody. A summary of primary and secondary antibodies and dilutions used is included in Table 3. After overnight incubation at $4{ }^{\circ} \mathrm{C}$ in a humidified chamber the sections were rinsed in TBS with $0.1 \%$ Tween $20(\mathrm{v} / \mathrm{v})$. The antigens were visualized using corresponding biotinylated secondary antibody $(1: 300,1.5 \mathrm{~h}$ at room temperature (RT); Vector Laboratories, Burlingame, CA, USA), avidin-biotin-peroxidase complex (1:100, $40 \mathrm{~min}$ at RT; StreptABComplex-HRP, Dako A/S, Glostrup, Denmark), and $3,3^{\prime}$-diaminobenzidine (DAB, Sigma-Aldrich) as a

Table 2 Details of TaqMan Gene Expression Assay used for real-time PCR analysis.

\begin{tabular}{lll}
\hline Gene & Description & Assay ID \\
\hline MKI67 & Ki-67 & Ss03389401_m1 \\
TNF & Tumor necrosis factor $\alpha$ (TNF $\alpha)$ & Ss03391317_g1 \\
GATA4 & GATA4 & Ss03383805_u1 \\
CDH1 & E-cadherin & Ss03377287_u1 \\
GAPDH & Glyceraldehyde-3-phosphate dehydrogenase (GAPDH) & Ss03375286_u1 \\
\hline
\end{tabular}


Table 3 The primary and secondary antibodies used in immunohistochemistry.

\begin{tabular}{lllc}
\hline Antibody & Origin & Clonality, Source & Dilution \\
\hline Ki-67 & Mouse & Monoclonal, Dako (cat\# M7240) & $1: 75$ \\
GATA4 & Goat & Polyclonal, Santa Cruz Biotechnology (cat\#sc-1237) & $1: 2000$ \\
E-cadherin & Rabbit & Polyclonal, Abcam (cat\# ab15148) & $1: 100$ \\
\hline
\end{tabular}

chromogen-staining substrate. The sections were dehydrated and mounted under glass with DPX. Negative control reactions included sections incubated with blocking serum containing nonspecific IgG instead of primary antibody and processed as described earlier. All slides except those of GATA4 identification, were counterstained with Mayer's hematoxylin. Selected sections were photographed using the Nikon Eclipse 80i microscope and the Nikon Digital Sight DS-Fi1 camera (Nikon, Japan) with corresponding software.

\section{Immunohistochemical staining evaluation}

\section{$K i-67$}

Cell scoring was performed on all Ki-67 immunohistochemical results and expressed as a percentage \pm S.E.M. The percentage was determined by the number of Ki-67-positive cells among the total number of counted cells in EN per section. The counting was performed in each field using a microscope with a $\times 40$ objective and a cell counter. Slides were coded and counted blindly by two independent investigators and the average of two readings was taken.

\section{GATA4 and E-cadherin}

In order to estimate the intensity of GATA4 and E-cadherin immunohistochemical staining the captured digital images ( $\times 20$ objective) for at least six different sections from each investigated animal were examined using the public domain Imagel software (National Institute of Health, Bethesda, MD, USA). The intensity of staining was expressed as relative optical density (ROD) and was calculated using the following formula:

$$
\begin{aligned}
\mathrm{ROD} & =\mathrm{OD}_{\text {specimen }} / \mathrm{OD}_{\text {background }} \\
& =\log \left(\mathrm{GL}_{\text {blank }} / \mathrm{GL}_{\text {specimen }}\right) / \log \left(\mathrm{GL}_{\text {blank }} / \mathrm{GL}_{\text {background }}\right),
\end{aligned}
$$

where GL means a gray level for stained area (specimen) and unstained area (background) and blank means a gray level measured after removing the slide from the light path (Smolen 1990). Results are reported as an overall mean ( \pm S.E.M.).

\section{TUNEL staining and quantitative analysis}

Apoptotic cells in ovarian sections were detected using TUNEL. The ApopTag Plus Peroxidase In Situ Apoptosis Detection Kit (Chemicon International, Melbourne, Australia) was used. The protocol of the manufacturer was followed. Briefly after deparaffinization and rehydration the slides were washed (5 min) in PBS (1.0 mM, pH 7.4) and thereafter sections were pretreated with proteinase $\mathrm{K}$ solution $(10 \mu \mathrm{g} / \mathrm{ml}$, Promega Corporation) for $15 \mathrm{~min}$ at RT. Next sections were rinsed in PBS, immersed in $3 \% \mathrm{H}_{2} \mathrm{O}_{2}$ in methanol $(\mathrm{v} / \mathrm{v})$ for $10 \mathrm{~min}$ at RT to quench endogenous peroxidase activity and then transferred into equilibration buffer. After $10 \mathrm{~min}$ at RT in a humidified chamber, working strength TdT enzyme was added to positive sections, while negative control sections received PBS. Plastic coverslips were added and slides were placed in a humidified chamber at $37^{\circ} \mathrm{C}$ for $1 \mathrm{~h}$. The slides were then washed with stop/wash buffer for $10 \mathrm{~min}$ at RT followed by a 5 min PBS wash. Excess of liquid was tapped off and the anti-digoxigenin conjugate was applied directly to the specimens and incubated for $30 \mathrm{~min}$ at RT in a humidified chamber. After rinsing in PBS (10 min) apoptotic cells were visualized with the addition of DAB solution for 8-10 min. A negative control was performed without active TdT enzyme but including proteinase $\mathrm{K}$ digestion to control for nonspecific incorporation of nucleotides or for nonspecific binding of enzyme conjugate. All the sections were counterstained using Mayer's hematoxylin next dehydrated through a graded series of ethanol and mounted under glass with DPX.

Apoptotic cell scoring was performed on all slides from control and treated ovaries. The number of TUNEL-positive cells with regard to the total number of counted oogonia/oocytes per section and was expressed as a percentage \pm S.E.M. The counting was performed in each field using a microscope with a $\times 40$ objective and a cell counter. Slides were coded and counted blindly by two independent investigators and the average of two readings was taken.

\section{Statistical analysis}

Statistical analysis was performed using Statistica 10 program (StatSoft, Inc., Tulsa, OK, USA). Differences between control and flutamide-treated groups assessed using the MannWhitney $U$ test. The data were statistically evaluated with significance at $* P<0.5, * * P<0.01,{ }^{* * *} P<0.001$.

\section{Declaration of interest}

The authors declare that there is no conflict of interest that could be perceived as prejudicing the impartiality of the research reported.

\section{Funding}

This work was supported by the Ministry of Science and Higher Education, grant No. N N303 596539 (to M Slomczynska).

\section{Acknowledgements}

The authors would like to thank Prof. Teresa Szklarzewicz (Department of Developmental Biology and Morphology of Invertebrates, Institute of Zoology, Jagiellonian University, 
Krakow, Poland) for providing imaging facilities. M DurlejGrzesiak is supported by funding from Jagiellonian University within the SET project. The project is co-financed by the European Union within the European Social Fund.

\section{References}

Abbott DH, Dumesic DA, Eisner JR, Colman RJ \& Kemnitz JW 1998 Insights into the development of polycystic ovary syndrome (PCOS) from studies of prenatally androgenized female rhesus monkeys. Trends in Endocrinology and Metabolism 9 62-67. (doi:10.1016/S1043-2760 (98)00019-8)

Abbott DH, Dumesic DA \& Franks S 2002 Developmental origin of polycystic ovary syndrome - a hypothesis. Journal of Endocrinology 174 1-5. (doi:10.1677/joe.0.1740001)

Aitken RJ, Findlay JK, Hutt KJ \& Kerr JB 2011 Apoptosis in the germ line. Reproduction 141 139-150. (doi:10.1530/REP-10-0232)

Bielanska-Osuchowska Z 2006 Oogenesis in pig ovaries during the prenatal period: ultrastructure and morphometry. Reproductive Biology 6 161-193.

de Boo HA \& Harding JE 2006 The developmental origins of adult disease (Barker) hypothesis. Australian \& New Zealand Journal of Obstetrics \&Gynaecology 46 4-14. (doi:10.1111/j.1479-828X.2006.00506.x)

Burek M, Duda M, Knapczyk K, Koziorowski M \& Słomczyńska M 2007 Tissue-specific distribution of the androgen receptor (AR) in the porcine fetus. Acta Histochemica 109 358-365. (doi:10.1016/j.acthis.2007. 03.003)

Carruba G, Miceli D, D'Amico D, Farrugio R, Comito L, Montesanti A, Polito L \& Castagnetta LA 1995 Sex steroids up-regulates E-cadherin expression in hormone-responsive LNCaP human prostate cancer cells. Biochemical and Biophysical Research Communications 212 624-631. (doi:10.1006/bbrc.1995.2015)

Ding W, Wang W, Zhou B, Zhang W, Huang P, Shi F \& Taya K 2010 Formation of primordial follicles and immunolocalization of PTEN, PKB and FOXO3A proteins in the ovaries of fetal and neonatal pigs. Journal of Reproduction and Development 56 162-168. (doi:10.1262/ jrd.09-094H)

Drummond AE 2006 The role of steroids in follicular growth. Reproductive Biology and Endocrinology 4 16-26. (doi:10.1186/1477-7827-4-16)

Durlej M, Knapczyk-Stwora K, Duda M, Galas J \& Slomczynska M 2011a The expression of FSH receptor (FSHR) in the neonatal porcine ovary and its regulation by flutamide. Reproduction in Domestic Animals 46 377-384. (doi:10.1111/j.1439-0531.2010.01673.x)

Durlej M, Kopera I, Knapczyk-Stwora K, Hejmej A, Duda M, Koziorowski M, Slomczynska M \& Bilińska B 2011b Connexin43 gene expression in male and female gonads of porcine offspring following in utero exposure to an antiandrogen, flutamide. Acta Histochemica 113 6-12. (doi:10.1016/j.acthis.2009.07.001)

Durlej M, Knapczyk-Stwora K \& Slomczynska M 2012 Prenatal and neonatal flutamide administration increases proliferation and reduces apoptosis in large antral follicles of adult pigs. Animal Reproduction Science 132 58-65. (doi:10.1016/j.anireprosci.2012.04.001)

Epifano O \& Dean J 2002 Genetic control of early folliculogenesis in mice. Trends in Endocrinology and Metabolism 13 169-173. (doi:10.1016/ S1043-2760(02)00576-3)

Foecking EM, Szabo M, Schwartz NB \& Levine JE 2005 Neuroendocrine consequences of prenatal androgen exposure in the female rat: absence of luteinizing hormone surges, suppression of progesterone receptor gene expression, and acceleration of the gonadotropin-releasing hormone pulse generator. Biology of Reproduction 72 1475-1483. (doi:10.1095/biolreprod.105.039800)

Forsdike RA, Hardy K, Bull L, Stark J, Webber LJ, Stubbs S, Robinson JE \& Franks S 2007 Disordered follicle development in ovaries of prenatally androgenized ewes. Journal of Endocrinology 192 421-428. (doi:10.1677/joe.1.07097)

Foster PM \& Harris MW 2005 Changes in androgen-mediated reproductive development in male rat offspring following exposure to a single oral dose of flutamide at different gestational ages. Toxicological Sciences 85 1024-1032. (doi:10.1093/toxsci/kfi159)
Garrett WM \& Guthrie HD 1999 Expression of $\mathrm{BCl}-2$ and $3-\beta$ hydroxysteroid dehydrogenase protein during oocyte and follicle development in fetal post-natal pig ovaries. Reproduction, Fertility, and Development 11 163-170. (doi:10.1071/RD00003)

Heikinheimo M, Ermolaeva M, Bielinska M, White RA \& Wilson DB 1997 Expression and hormonal regulation of transcription factors GATA-4 and GATA-6 in the mouse ovary. Endocrinology 138 3505-3514. (doi:10.1210/en.138.8.3505)

Ing NH 2005 Steroid hormones regulate gene expression posttranscriptionally by altering the stabilities of messenger RNAs. Biology of Reproduction 72 1290-1296. (doi:10.1095/biolreprod.105.040014)

Knapczyk-Stwora K, Sternak M, Durlej M \& Slomczynska M 2011 Immunolocalization of cytochrome P450 17 $\alpha$-hydroxylase/c17-20 lyase in the ovary of pregnant pigs and fetal gonads. Reproductive Biology 11 71-82. (doi:10.1016/S1642-431X(12)60046-1)

Livak KJ \& Schmittgen TD 2001 Analysis of relative gene expression data using real-time quantitative PCR and the $2^{-\Delta \Delta C t}$ method. Methods 25 402-408. (doi:10.1006/meth.2001.1262)

Mackay S, Nicholson CL, Lewis SP \& Brittan M 1999 E-cadherin in the developing mouse gonad. Anatomy and Embryology 200 91-102. (doi:10.1007/s004290050263)

McCoard SA, Wise TH, Fahrenkrug SC \& Ford JF 2001 Temporal and spatial localization patterns of Gata4 during porcine gonadogenesis. Biology of Reproduction 65 366-374. (doi:10.1095/biolreprod65.2.366)

Morrison LJ \& Marcinkiewicz JL 2002 Tumor necrosis factor $\alpha$ enhances oocyte/follicle apoptosis in the neonatal rat ovary. Biology of Reproduction 66 450-457. (doi:10.1095/biolreprod66.2.450)

Nightingale J, Chaudhary KS, Abel PD, Stubbs AP, Romanska HM, Mitchell SE, Stamp GWH \& Lalani EN 2003 Ligand activation of the androgen receptor downregulates E-cadherin-mediated cell adhesion and promotes apoptosis of prostatic cancer cells. Neoplasia 5 347-361.

Ortega HH, Salvetti NR \& Padmanabhan V 2009 Developmental programming: prenatal androgen excess disrupts ovarian steroid receptor balance. Reproduction 137 865-877. (doi:10.1530/REP-08-0491)

Ryan PL, Valentine AF \& Bagnell CA 1996 Expression of epithelial cadherin in the developing and adult pig ovary. Biology of Reproduction $\mathbf{5 5}$ 1091-1097. (doi:10.1095/biolreprod55.5.1091)

Sarraj MA \& Drummond AE 2012 Mammalian foetal ovarian development: consequences for health and disease. Reproduction 143 151-163. (doi:10.1530/REP-11-0247)

Smolen AJ 1990 Image analytic techniques for quantification of immunocytochemical staining in the nervous system. In Methods in Neurosciences, pp 208-229. Ed PM Conn. New York: Academic Press.

Steckler T, Manikkam M, Inskeep EK \& Padmanabhan V 2007 Developmental programming: follicular persistence in prenatal testosteronetreated sheep is not programmed by androgenic action of testosterone. Endocrinology 148 3532-3540. (doi:10.1210/en.2007-0339)

Stoop H, Honecker F, Cools M, deKrijger R, Bokemeyer C \& Looijenga LH] 2005 Differentiation and development of human female germ cells during prenatal gonadogenesis: an immunohistochemical study. Human Reproduction 20 1466-1476. (doi:10.1093/humrep/deh800)

Sullivan SD \& Moenter SM 2004 Prenatal androgens alter GABAergic drive to gonadotropin-releasing hormone neurons: implications for a common fertility disorder. PNAS 101 7129-7134. (doi:10.1073/pnas. 0308058101)

Sweeney T 2002 Is exposure to endocrine disrupting compounds during fetal/post-natal development affecting the reproductive potential of farm animals? Domestic Animal Endocrinology 23 203-209. (doi:10.1016/ S0739-7240(02)00157-1)

Tevell A, Lennernas H, Jonsson M, Norlin M, Lennernas B, Bondesson U \& Hedeland M 2006 Flutamide metabolism in four different species in vitro and identification of flutamide metabolites in human patient urine by high performance liquid chromatography/tandem mass spectrometry. Drug Metabolism and Disposition 34 982-992. (doi:10.1124/dmd.105. 008516)

Uzumcu M \& Zachow R 2007 Developmental exposure to environmental endocrine disruptors: consequences within the ovary and on female reproductive function. Reproductive Toxicology 23 337-352. (doi:10.1016/j.reprotox.2006.10.006)

Vendola KA, Zhou J, Adesanya OO, Weil SJ \& Bondy CA 1998 Androgens stimulate early stages of follicular growth in the primate ovary. Journal of Clinical Investigation 101 2622-2629. (doi:10.1172/JCI2081) 
Walters KA, Allan CM \& Handelsman DJ 2008 Androgen actions and the ovary. Biology of Reproduction 78 380-389. (doi:10.1095/biolreprod. 107.064089)

Wang C \& Roy SK 2010 Expression of E-cadherin and N-cadherin in perinatal hamster ovary: possible involvement in primordial follicle formation and regulation by follicle-stimulating hormone. Endocrinology 151 2319-2330. (doi:10.1210/en.2009-1489)

Williams K, McKinnell C, Saunders PT, Walker M, Fisher JS, Turner KJ, Atanassova N \& Sharpe M 2001 Neonatal exposure to potent and environmental oestrogens and abnormalities of the male reproductive system in the rat: evidence for importance of the androgen-oestrogen balance and assessment of the relevance to man. Human Reproduction Update 7 236-247. (doi:10.1093/humupd/7.3.236)
Yang YL, Zhang CP, Li L, Huang L, Ji SY, Lu CL, Fan CH, Cai H, Ren Y, Hu ZY et al. 2010 Testosterone induces redistribution of forkhead box-3a and down-regulation of growth and differentiation factor 9 messenger ribonucleic acid expression at earl stage of mouse folliculogenesis. Endocrinology 151 774-782. (doi:10.1210/en.2009-0751)

Received 20 June 2012

First decision 23 July 2012

Revised manuscript received 14 January 2013

Accepted 15 January 2013 